\title{
Abrupt Transition to Strong Superrotation Driven by Equatorial Wave Resonance in an Idealized GCM
}

\section{Citation}

Arnold, Nathan P., Eli Tziperman, and Brian Farrell. 2012. "Abrupt Transition to Strong Superrotation Driven by Equatorial Wave Resonance in an Idealized GCM." Journal of the Atmospheric Sciences 69 (2): 626-40. https://doi.org/10.1175/jas-d-11-0136.1.

\section{Permanent link}

http://nrs.harvard.edu/urn-3:HUL.InstRepos:41384981

\section{Terms of Use}

This article was downloaded from Harvard University's DASH repository, and is made available under the terms and conditions applicable to Other Posted Material, as set forth at http:// nrs.harvard.edu/urn-3:HUL.InstRepos:dash.current.terms-of-use\#LAA

\section{Share Your Story}

The Harvard community has made this article openly available.

Please share how this access benefits you. Submit a story.

Accessibility 


\title{
Abrupt Transition to Strong Superrotation Driven by Equatorial Wave Resonance in an Idealized GCM
}

\author{
NATHAN P. ARNOLD \\ Department of Earth and Planetary Sciences, Harvard University, Cambridge, Massachusetts \\ ELI TZIPERMAN AND BRIAN FARRELL \\ Department of Earth and Planetary Sciences, and School of Engineering and Applied Sciences, \\ Harvard University, Cambridge, Massachusetts
}

(Manuscript received 16 May 2011, in final form 16 August 2011)

\begin{abstract}
Persistent superrotation is seen in the atmospheres of other terrestrial bodies (Venus, Titan) but not in that of present Earth, which is distinguished by equatorial easterlies. Nevertheless, superrotation has appeared in numerical simulations of Earth's atmosphere, from two-layer models to multilevel comprehensive GCMs. Simulations of warm climates that generate enhanced tropical convective variability seem particularly prone to superrotation, which has led to hypotheses that the warmer atmospheres of the early Pliocene and Eocene may have been superrotating, and that the phenomenon may be relevant to future climate projections.

This paper considers a positive feedback leading to superrotation based on an equatorial wave resonance that occurs in a westerly background flow. The authors present simulations with an idealized multilevel GCM forced with a zonally varying equatorial heating, which show abrupt transitions to strongly superrotating states. Linear shallow water theory is used to show that these transitions occur as the superrotating jet velocity approaches the phase speed of free equatorial Rossby wave modes, leading to a resonant amplification of the response to eddy heating and its associated equatorward momentum flux. The resonance and transition are most prominent in simulations where the meridional temperature gradient has been reduced, and hysteresis behavior is seen when the gradient is eliminated completely. No evidence is found in these simulations for the midlatitude wave feedback believed to drive abrupt transitions in two-layer models, and there is only a minor role for the axisymmetric feedback based on vertical advection by the Hadley circulation.
\end{abstract}

\section{Introduction}

Superrotation refers to an atmospheric circulation with persistent zonal-mean westerly winds over the equator. Such a state is relatively common in our solar system, being observed in the gas giants Jupiter and Saturn, as well as the atmospheres of Venus and Titan. Earth's atmosphere is also known to superrotate, but only in the stratosphere during the westerly phase of the quasi-biennial oscillation. It has been suggested that superrotation may be excited in a global warming scenario (Held 1999; Pierrehumbert 2000) and that it may explain the Pliocene "permanent El Niño" inferred from proxy observations (Tziperman and Farrell

Corresponding author address: Nathan Arnold, Harvard University, 24 Oxford St., Cambridge, MA 02138.

E-mail: narnold@fas.harvard.edu
2009). It is therefore of significant interest to understand the physical mechanisms that may lead to a superrotating state.

Hide (1969) showed that prograde (westerly) equatorial winds are impossible without large-scale, up-gradient eddy transport of angular momentum, which is primarily accomplished in Earth's atmosphere by Rossby waves (e.g., Held 1999). Because Rossby waves act to accelerate their latitude of origin, superrotation must generally involve an equatorial wave source. Organized tropical convection can provide such a source and has been associated with the onset of superrotation in previous studies (Lee 1999; Caballero and Huber 2010; Grabowski 2004). Superrotation will occur in general when the momentum convergence due to equatorial waves exceeds the divergence due to other phenomena, particularly the seasonal cross-equatorial flow of the Hadley cell (Kraucunas and Hartmann 2005). 
Persistent tropospheric superrotation has appeared in previous numerical simulations, and it is useful here to distinguish between those that develop superrotation gradually and those with transitions that are "abrupt." In the former case, the atmosphere responds to a continuous change in external parameter (e.g., $\mathrm{CO}_{2}$ concentration) by varying continuously from one equilibrium state to the next, while the latter case is marked by threshold behavior; a small change in external parameter may trigger positive feedbacks and lead to a qualitatively different climatology.

In this paper, we study a novel positive feedback based on a resonant Rossby wave response to equatorial heating. The Rossby wave response to stationary equatorial forcing is evanescent unless the mean flow speed is equal and opposite to the free Rossby wave phase speed. This leads to a positive feedback between the waves and mean flow: as a westerly background zonal wind approaches the phase speed of the free equatorial Rossby wave, the waves will amplify. This amplification leads to stronger equatorward momentum flux, enhancing the mean westerlies and thereby further amplifying the Rossby waves. A resonance is implied, as the maximum wave amplification and mean flow acceleration occur when the westerly flow speed is exactly equal to the westward Rossby wave phase speed.

One scenario in which this feedback may be relevant is suggested by the observed annual mean pattern of geopotential height and horizontal flow at $150 \mathrm{mb}$ around the Maritime Continent, a broad region of very active convection (e.g., Fig. 4 of Dima et al. 2005). This pattern resembles the theoretical Gill solution for the upper tropospheric response to an isolated heat source, consisting of a pair of Rossby gyres to the west of the heating maximum and a Kelvin wave response to the east. This stationary wave pattern is responsible for most of the equatorward momentum flux in the modern tropics. Both the wave pattern and its momentum transport would be expected to amplify in the presence of a westerly background wind, potentially leading to large changes in local climate, or even the establishment of superrotation on a global scale.

We demonstrate that this resonance occurs in a multilevel idealized GCM. The idealized configuration allows a straightforward comparison with shallow water theory, which confirms the physics described above. We force the idealized GCM with a zonally varying equatorial heating and show that a superrotating jet can develop via a bifurcation as the heating rate is increased, jumping from a relatively weak to a much stronger state, with significant changes to the global circulation.

The earliest examples of abrupt transitions were seen in two-layer primitive equation models that spontaneously jumped from a conventional circulation to a superrotating one (Held and Suarez 1978). Later studies employing equatorial eddy heating (Suarez and Duffy 1992; Saravanan 1993) explained this behavior in terms of a competition between extratropical and equatorial Rossby wave transport of angular momentum. In a conventional circulation, baroclinic waves generated in midlatitudes may propagate equatorward until they are absorbed near critical layers in the subtropics, where their phase speed is equal to the mean zonal flow (Randel and Held 1991). This leads to angular momentum flux from the subtropics to midlatitudes. In these two-layer models, the conventional circulation is maintained by a negative feedback; small westerly perturbations to the equatorial winds will move critical layers equatorward, making the baroclinic wave drag more efficient and restoring the conventional easterly flow.

However, for sufficiently large westerly perturbations, the critical layers may disappear altogether, leaving the tropics effectively transparent to baroclinic waves. In this scenario, the only torque associated with baroclinic waves results from their frictional dissipation as they propagate across the tropics and is much weaker than the torque provided by critical layer absorption. This asymmetry allows for abrupt transitions in equatorial wind strength when the baroclinic wave feedback changes sign.

Shell and Held (2004) demonstrated that abrupt transitions and multiple equilibria are also possible in an axisymmetric framework. The positive feedback here is based on vertical advection by the Hadley cell, which brings quiescent surface air into the upper troposphere and serves as a source of drag on any superrotating flow. However, the greater angular momentum associated with a superrotating flow implies a reduced Hadley cell mass flux, which in turn implies a reduction in drag.

Williams $(2003,2006)$ varied the latitude of maximum baroclinity in an idealized multilevel primitive equation model and showed that when this latitude is less than $20^{\circ}$ from the equator, a barotropic instability on the equatorward side of the eddy-driven jet leads to westerly equatorial winds. Williams showed that the boundary between conventional and superrotating equilibria is quite narrow in parameter space and suggested that bifurcations should be possible.

Abrupt transitions are typically not seen in "comprehensive" multilevel GCMs, which include subgridscale physics parameterizations, seasonal cycles, and other realistic features. However, gradual transitions to superrotation have been seen, particularly in simulations of global warming scenarios or warm paleoclimates (Huang et al. 2001; Caballero and Huber 2010). In some cases, these are accompanied by an increase in tropical 
intraseasonal variability (Lee 1999; Caballero and Huber 2010), which may serve as an equatorial wave source. If the superrotation in these cases is indeed driven by changes in organized convection, then it may depend on particular parameterization schemes, a poorly constrained component of atmospheric models. Note that the positive feedbacks discussed above-that is, those based on Rossby wave critical layers, the Hadley circulation, and barotropic instability-are all determined solely by the primitive equations, and should thus be present to some degree in every GCM. The scarcity of abrupt transitions in comprehensive models suggests either a lack of the sustained westerly perturbations required to activate positive feedbacks, a stronger role for negative feedbacks, or an atmosphere dominated by "stochastic" variability that prevents a sustained feedback loop.

This paper is organized as follows: The model and forcing are described in section 2 . In section 3 we present idealized simulations illustrating the feedback and bifurcation. A quantitative explanation of the feedback based on linear shallow water theory is offered in section 4. Section 5 explores the feedback in more realistic climates, and our conclusions and further discussion are in section 6.

\section{Model and experimental setup}

\section{a. Model description}

We use the National Center for Atmospheric Research (NCAR) Community Atmosphere Model (CAM) in the idealized configuration based on Held and Suarez (1994). This consists of the dry primitive equations for an ideal gas on a rotating sphere and neglects topography, moisture, and radiative and convective processes. The effect of the omitted processes on the model climate is crudely represented by a Newtonian relaxation of temperatures toward a prescribed "radiative-convective" equilibrium profile. Surface momentum exchange in the boundary layer is represented by a linear Rayleigh friction. $\mathrm{Nu}-$ merical stability is maintained with a weak horizontal biharmonic diffusion, and vertical diffusion is neglected. This idealized configuration is advantageous in that it captures the fundamental physics of the large-scale circulation while remaining simple enough that model output may be easily compared with theory.

We modify the prescribed temperature profile to allow for a reduced - and in some cases, eliminated - meridional temperature gradient, which serves to modulate the strength of the Hadley cell and the eddies associated with midlatitude baroclinicity. This simplification reveals the feedback and bifurcation that are the focus of this paper. The modified equilibrium temperature profile is of the form

$$
\begin{aligned}
T_{\mathrm{eq}}(p, \phi)= & {\left[315 \mathrm{~K}-\Delta T \sin ^{2} \phi-(\Delta \theta)_{z} \log \left(\frac{p}{p_{0}}\right) \cos ^{2} \phi\right] } \\
& \times\left(\frac{p}{p_{0}}\right)^{R / c_{p}},
\end{aligned}
$$

where the equator-to-pole temperature difference $\Delta T$ is varied from 0 to $60 \mathrm{~K}$, and the $\cos ^{2} \phi$ factor is replaced by 1 in the case $\Delta T=0 \mathrm{~K}$ in order to eliminate the temperature gradient associated with a meridionally varying lapse rate. Similar to studies in the Matsuno-Gill framework (e.g., Gill 1980), setting $\Delta T=0 \mathrm{~K}$ isolates the equatorial dynamics and simplifies the analysis, but it should be kept in mind that this also removes dominant processes from the equatorial momentum balance, in particular the momentum transport by midlatitude waves and the seasonal cross-equatorial flow of the Hadley circulation that maintains the easterlies near the present tropical tropopause (Lee 1999). We address this issue in section 5.

The lack of vertical diffusion is a serious deficiency when studying an equatorial jet, as this leaves only resolved, large-scale advection to represent the small-scale convective plumes and broad subsidence that make up vertical motion in the real tropical troposphere. It has been noted by Held (1999) that superrotation can generally be eliminated in idealized models by increasing the vertical diffusion. Although cumulus friction (convective momentum transport) is a secondary term in the presentday equatorial momentum balance, this is partly a result of weak vertical shear, and it may play a larger role in a superrotating scenario. Gravity wave drag is also known to play a small but significant role (Huang et al. 1999), but is not included in the model.

The simulations presented here are based on the CAM3.1 spectral dynamical core with T42 truncation (a horizontal resolution of roughly $2.8^{\circ} \times 2.8^{\circ}$ ) and 26 vertical levels. Some simulations were repeated with the same dynamical core at a higher resolution (T85, roughly $1.4^{\circ} \times 1.4^{\circ}$ ), or with the CAM4.0 finite volume dynamical core, at $1.9^{\circ} \times 2.5^{\circ}$ resolution. We found no significant differences with either higher resolution or the alternative dynamical core.

\section{b. Prescribed eddy forcing}

The appearance of superrotation in many numerical studies has coincided with increases in organized tropical convection (Lee 1999; Caballero and Huber 2010), and it is generally accepted that large-scale variations in convective heating can act as a Rossby wave source and lead to equatorward momentum fluxes. To mimic the effect of latent heating in our dry model, we add a diabatic heating term to the model's thermodynamic tendency equation, of the form 
$Q_{\mathrm{CAM}}=Q_{0} \cos \left[\frac{k\left(\lambda-c_{f} t\right)}{2 \pi a}\right] \exp \left(-\frac{\phi^{2}}{\Delta \phi^{2}}\right) \sin \left(\pi \frac{p-p_{t}}{p_{b}-p_{t}}\right)$,

similar to that used by Kraucunas and Hartmann (2005). Here $p_{t}$ is pressure at the tropopause, $p_{b}$ pressure at the top of the boundary layer, $k$ the zonal wavenumber, and $c_{f}$ a zonal translation speed. The heating is Gaussian in latitude, sinusoidal in longitude, approximates the first baroclinic mode in the vertical, and is limited to the free troposphere, between $p_{b}=800 \mathrm{mb}$ and $p_{t}=200 \mathrm{mb}$. In this paper we use $\Delta \phi=10^{\circ}$, roughly the equatorial deformation radius, and vary $Q_{0}, k$, and $c_{f}$.

For reference, the zonally varying component of the time-mean tropical latent heating can be approximated by $k=2, \Delta \phi=15^{\circ}, c=0 \mathrm{~m} \mathrm{~s}^{-1}$, and $Q_{0}=1 \mathrm{~K} \mathrm{day}^{-1}$ (Schumacher et al. 2004). The Madden-Julian oscillation (MJO) might be thought of as $k=1, \Delta \phi=15^{\circ}, c_{f}=$ $5 \mathrm{~m} \mathrm{~s}^{-1}$, and $Q_{0}=2 \mathrm{~K} \mathrm{day}^{-1}$ (Kiladis et al. 2005). The simulations presented here employ heating rates well within these bounds, never exceeding $Q_{0}=0.9 \mathrm{~K} \mathrm{day}^{-1}$. However, the forcing could be more effective than its magnitude suggests because it is so spatially coherent and continuous in time.

We found that the transition to superrotation could be triggered with nearly any zonally asymmetric heating. Related simulations with a prescribed momentum forcing also produced a bifurcation. The results thus appear insensitive to the form of equatorial forcing, so long as some equatorial wave source is present.

\section{Resonance and abrupt transition with no meridional temperature gradient}

Although the resonance and bifurcation analyzed below occur for a wide range of parameters, we begin with the case in which they are most clearly illustrated, with the pole-to-equator temperature difference $\Delta T$ set to zero. The model is initialized from rest and allowed to reach equilibrium with wavenumber-2 heating of constant $Q_{0}=0.1 \mathrm{~K} \mathrm{day}^{-1}$. The direct response to the heating takes three forms: a first baroclinic structure in the equatorial troposphere, similar to the classical MatsunoGill response (Gill 1980); a weak vertically propagating signal, confined to the tropics; and weak polewardpropagating Rossby waves with an equivalent barotropic structure. This is consistent with the work of Salby and Garcia (1987), who studied the response to equatorial heating in detail.

The zonal mean climate is shown in Fig. 1. Horizontal eddy momentum fluxes associated with the first baroclinic structure produce a weak westerly jet in the middle troposphere, and a small indirect circulation forms above the boundary layer in order to maintain thermal wind balance.

The heating is increased in increments of $0.1 \mathrm{~K} \mathrm{day}^{-1}$, and the model allowed to equilibrate at each level, until $Q_{0}=0.4 \mathrm{~K} \mathrm{day}^{-1}$. A time series of zonal velocity and horizontal eddy momentum convergence $-\overline{\partial_{y}\left(u^{\prime} v^{\prime}\right)}$ illustrates this sequence in Fig. 2. For $Q_{0}<0.4 \mathrm{~K}$ day $^{-1}$, the eddy momentum convergence scales approximately as $Q_{0}^{2}$, as one might expect in a linear regime where $u^{\prime}$ and $v^{\prime}$ both scale with $Q_{0}$.

After $Q_{0}$ is increased to $0.4 \mathrm{~K} \mathrm{day}^{-1}$, the eddy momentum convergence initially rises according to the $Q_{0}^{2}$ scaling, but then the system appears to pass a threshold; the momentum convergence increases rapidly, peaking at $0.21 \mathrm{~m} \mathrm{~s}^{-1}$ day $^{-1}$ before settling around $0.13 \mathrm{~m} \mathrm{~s}^{-1} \mathrm{day}^{-1}$, significantly higher than the 0.07 $\mathrm{m} \mathrm{s}^{-1}$ day $^{-1}$ expected from the $Q_{0}^{2}$ scaling. This increase is not balanced by other dominant terms in the momentum equation, particularly diffusion and vertical eddy fluxes, and the result is a jump in the mean zonal velocity from 2 to nearly $24 \mathrm{~m} \mathrm{~s}^{-1}$. We show below that this sharp increase in eddy momentum flux and zonal velocity is due to a bifurcation in the system, driven by interaction between the mean flow and the eddy field.

The system also shows moderate hysteresis: if the heating rate is subsequently reduced to $0.3 \mathrm{~K}_{\text {day }}{ }^{-1}$, the eddy momentum convergence drops sharply to near its prebifurcation level, and the jet begins to decelerate. However, as the jet velocity decreases, the eddy momentum convergence begins to rise again and the jet equilibrates at $19 \mathrm{~m} \mathrm{~s}^{-1}$, much higher than the equilibrium velocity for $Q_{0}=0.3 \mathrm{~K} \mathrm{day}^{-1}$ before the bifurcation. This behavior suggests a resonance-like feedback, with a resonant velocity between the pre- and postbifurcation velocities, 2 and $19 \mathrm{~m} \mathrm{~s}^{-1}$.

In the time series in Fig. 2, we define the jet velocity as the zonal-mean zonal wind averaged from $5^{\circ} \mathrm{S}$ to $5^{\circ} \mathrm{N}$ and between 200 and $400 \mathrm{mb}$, although the bifurcation is also evident when averaging the full vertical column. This pressure interval is chosen because it is the region of greatest eddy activity, and we show below that it is the local (upper tropospheric) winds that are most relevant to the eddy-driven feedback. Prior to the bifurcation, both eddy kinetic energy and the meridional eddy momentum flux are concentrated in two altitude ranges: above the heating between 200 and $400 \mathrm{mb}$, and below the heating between 600 and $700 \mathrm{mb}$. The postbifurcation increase in these quantities is centered entirely in the upper troposphere. Note that this vertical shift across the bifurcation is also reflected in the vertical structure of the jet (see Figs. 1a,b). 

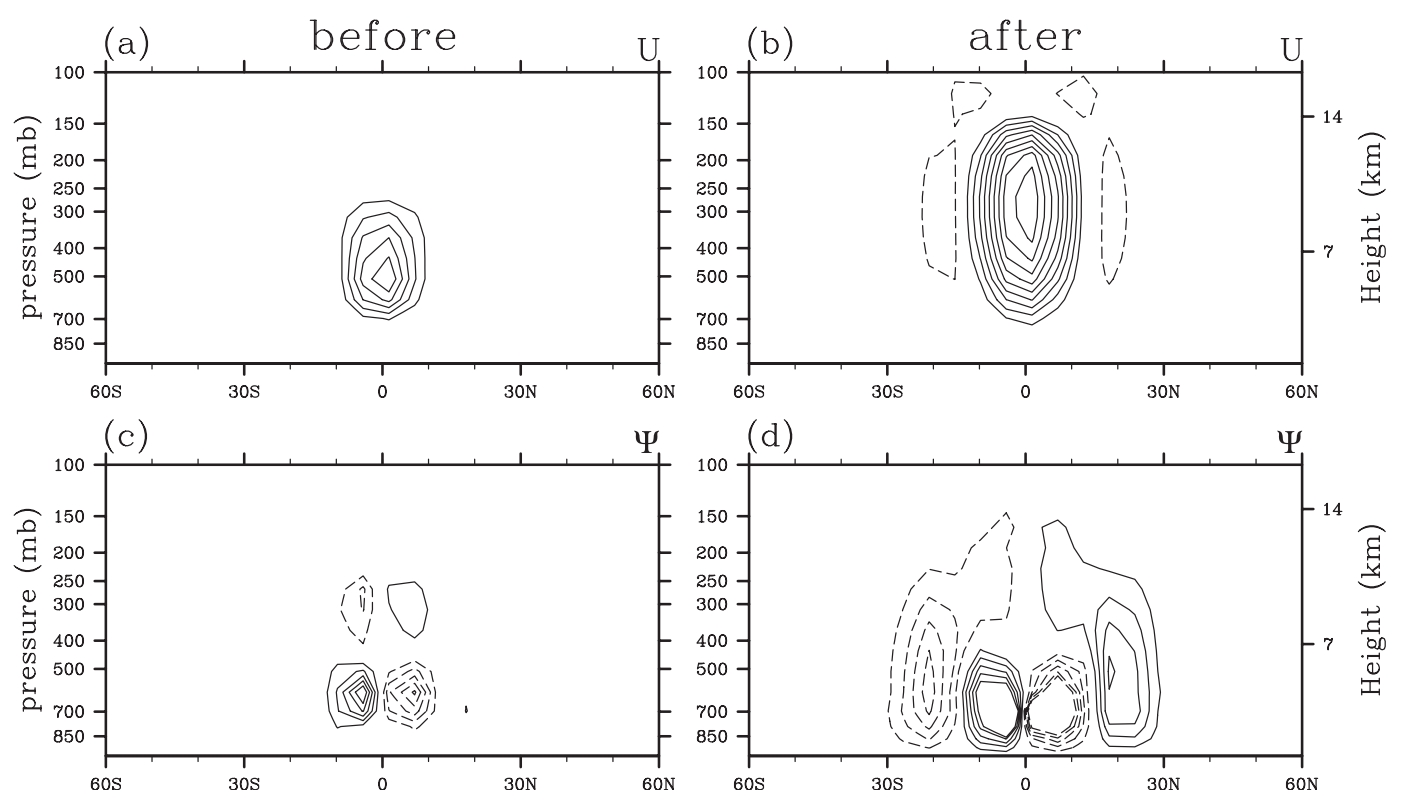

FIG. 1. Zonally averaged quantities, (a),(c) before and (b),(d) after the bifurcation, for simulations without meridional temperature gradient, $\Delta T=0 \mathrm{~K}$. All fields are in equilibrium with $Q_{0}=0.3 \mathrm{~K} \mathrm{day}^{-1}$, demonstrating multiple equilibria for this limited case. Contour intervals are (a) $1 \mathrm{~m} \mathrm{~s}^{-1}$, (b) $2.5 \mathrm{~m} \mathrm{~s}^{-1}$, and (c), (d) $0.5 \times 10^{9} \mathrm{~kg} \mathrm{~s}^{-1}$ ). Negative contours are dashed.

\section{Analysis of the wave-mean flow feedback and transition to superrotation}

We can gain some insight into the mechanism of the transition to superrotation by considering analytical solutions to the forced shallow water equations on an equatorial beta plane. Although the simulated jet has a nearly Gaussian meridional structure, explicitly taking this structure into account renders analytical solutions intractable. Instead we consider the response to forcing in the presence of a uniform background flow $U$, following the approach of Phlips and Gill (1987), in the hope that this approximation still captures the essential mechanism of the bifurcation. Showman and Polvani (2010) recently pointed out that the classical MatsunoGill system will not lead to superrotation due to an artificial cancellation between the horizontal and vertical eddy fluxes. This results from neglect of the momentum transport by the imposed mass/heat source. When this term is accounted for, vertical fluxes become smaller than horizontal fluxes and superrotation will develop. Consistent with their corrected model, the vertical eddy fluxes in our simulations are generally of opposite sign to the horizontal fluxes and smaller in magnitude. However, the temporal evolution of the vertical fluxes during transitions to superrotation varies significantly between simulations, so for simplicity we will focus on the horizontal fluxes. We believe this is consistent with our use of the shallow water framework only to gain physical insight into the wave-mean flow feedback.

We begin with the nondimensional steady-state shallow water equations:

$$
\begin{aligned}
U \partial_{x} u-\beta y v & =-\partial_{x} \phi-\kappa \nabla^{4} u, \\
U \partial_{x} v+\beta y u & =-\partial_{y} \phi-\kappa \nabla^{4} v, \\
U \partial_{x} \phi+\partial_{x} u+\partial_{y} \phi & =Q_{\mathrm{SW}} .
\end{aligned}
$$

The $\nabla^{4}$ hyperdiffusion term is included because of its appearance in CAM, and for a given simulation may be approximated as $-\epsilon u$, where $\epsilon=\kappa\left(k^{2}+l^{2}\right), k$ is the heating zonal wavenumber and $l$ is assumed to be $2 \pi$ over the deformation radius. This value is very near the coefficient of Rayleigh friction used in classical MatsunoGill studies (e.g., Gill 1980).

We assume a heat forcing given by

$$
Q_{\mathrm{SW}}=Q_{0} \cos (k x) \exp \left(-y^{2} / L_{d}^{2}\right)
$$

which excites only the Kelvin and $n=1$ planetary wave modes, and allows a simple Fourier transform in longitude, greatly simplifying the problem. The meridional structure can be found in terms of Hermite polynomials, following the well-known methodology outlined by Gill and others. Details for this case can be found in the appendix. 

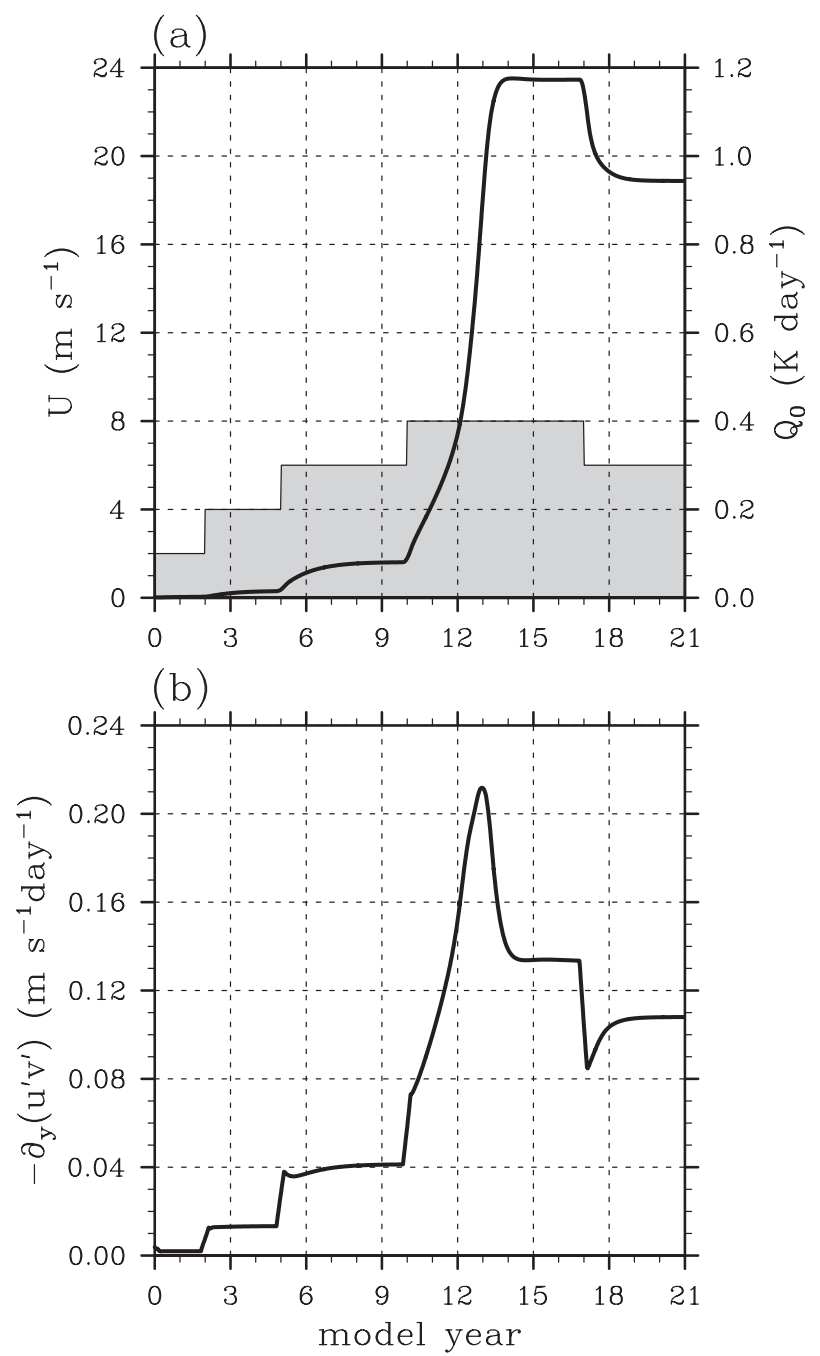

FIG. 2. Bifurcation of the equatorial jet in a simulation with no meridional temperature gradient, $\Delta T=0 \mathrm{~K}$. Time series of zonal mean quantities averaged between 200 and $400 \mathrm{mb}$ for the case of wavenumber $k=2$ heating with stepwise increasing heating rate $Q_{0}$. (a) Zonal velocity and heating rate $Q_{0}$; (b) equatorial eddy momentum convergence $-\partial_{y}\left(u^{\prime} v^{\prime}\right)$.

The gravity wave phase speed on which the solutions depend is a function of the equivalent depth, $h$, which we estimate from CAM in two ways. First, by direct calculation $h=(1 / g)(N / m)^{2}$, where $N$ is the buoyancy frequency and $m$ the vertical wavenumber of the imposed heating $(\pi / 12 \mathrm{~km})$. This method is complicated by the fact that $h$ varies significantly over the relevant altitudes: between 50 and $100 \mathrm{~m}$ for most of the troposphere, but from 100 to $600 \mathrm{~m}$ in the interval $150-250 \mathrm{mb}$, where the eddy momentum flux is largest. The second estimate is taken from the wavenumber-frequency power spectrum of $200-\mathrm{mb}$ zonal wind for a simulation without equatorial forcing, which shows enhanced power in bands associated with linear Kelvin and Rossby waves. After correcting for the Doppler shift by the mean zonal wind (estimated to be $-3 \mathrm{~m} \mathrm{~s}^{-1}$ ), the observed Kelvin wave speeds are most consistent with an equivalent depth of $150 \mathrm{~m}$, while the $n=1$ Rossby wave speeds are consistent with slightly larger depths of $150-250 \mathrm{~m}$. In the comparisons discussed below, we have used an equivalent depth $h=250 \mathrm{~m}$, which produces the best agreement between CAM and the shallow water theory. We acknowledge the somewhat arbitrary nature of this choice as a weak point in our analysis, but we believe it is consistent with the facts outlined above and permits a compelling simple model of the GCM behavior.

The nondimensional solution for the Kelvin mode is given by

$$
\begin{aligned}
u & =\frac{-Q_{0} \gamma}{6 \epsilon\left(1+\gamma^{2}\right)}[\gamma \cos (k x)+\sin (k x)]\left(y^{2}-3\right) \exp \left(-y^{2} / 4\right), \\
\phi & =\frac{-Q_{0} \gamma}{6 \epsilon\left(1+\gamma^{2}\right)}[\gamma \cos (k x)+\sin (k x)]\left(y^{2}-3\right) \exp \left(-y^{2} / 4\right),
\end{aligned}
$$

while the Rossby mode is given by

$$
\begin{aligned}
u= & \frac{-Q_{0} \gamma}{6 \epsilon\left(1+\gamma^{2}\right)}[\gamma \cos (k x)+\sin (k x)]\left(y^{2}-3\right) \exp \left(-y^{2} / 4\right), \\
v= & \left\{\frac{-4 Q_{0} \gamma}{3 \epsilon\left(1+\gamma^{2}\right)}[(U k+\gamma \epsilon) \cos (k x)\right. \\
& \left.+(\epsilon-U k \gamma) \sin (k x)]+Q_{0} \cos (k x)\right\} y \exp \left(-y^{2} / 4\right), \\
\phi= & \frac{-Q_{0} \gamma}{6 \epsilon\left(1+\gamma^{2}\right)}[\gamma \cos (k x)+\sin (k x)]\left(y^{2}+1\right) \exp \left(-y^{2} / 4\right) .
\end{aligned}
$$

where $\gamma=\epsilon / k(c+U), c$ being the appropriate wave phase speed (Kelvin or Rossby).

The significance of the solutions is best illustrated by the inviscid case $(\epsilon=0)$, in which the wave fields become proportional to $1 /(c+U)$. This implies a singularity when $U=-c$, directly analogous to the theoretical singularity involving topographic Rossby waves in the presence of a mean westerly wind (cf. Holton 2004). As $U \rightarrow-c_{R}$, the free Rossby mode becomes stationary relative to the heating and will amplify without bound in the absence of friction. In the presence of friction solutions reach a maximum amplitude when $U=-c_{R}$, which may be physically interpreted as a resonance.

The combined Kelvin and Rossby analytic solutions for $k=2$ are shown in Fig. 3 for $U=2 \mathrm{~m} \mathrm{~s}^{-1}$ and $U=$ $19 \mathrm{~m} \mathrm{~s}^{-1}$, along with 200-mb eddy fields from CAM, at $t=9 \mathrm{yr}$ and $t=20 \mathrm{yr}$ of the simulation shown in Fig. 2. The CAM eddy fields are defined as instantaneous 

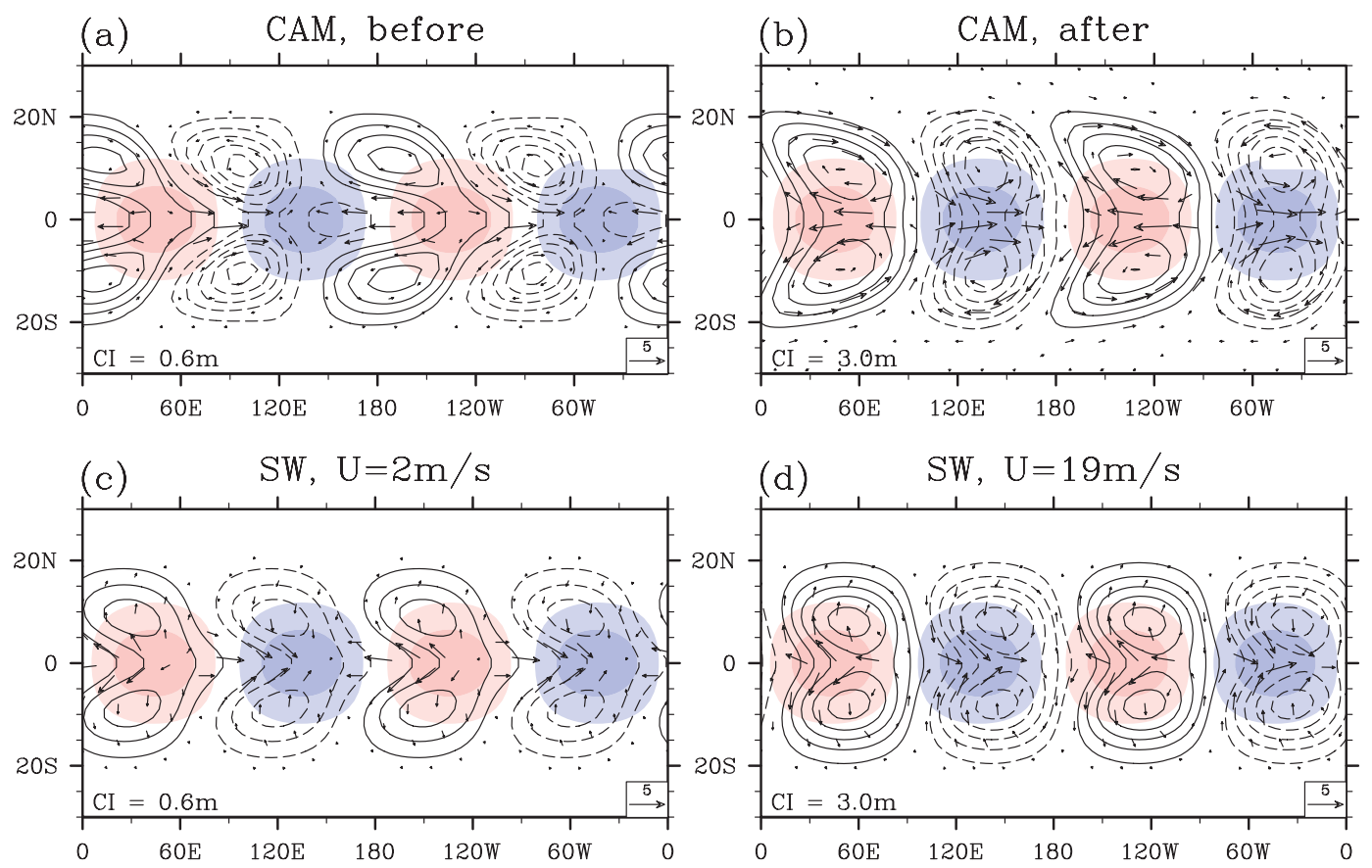

FIG. 3. Equilibrium eddy response (a),(c) before and (b),(d) after the bifurcation, with $Q_{0}=0.3 \mathrm{~K} \mathrm{day}^{-1}$, showing (a),(b) CAM 200-mb fields and (c),(d) shallow water solutions. Contours indicate geopotential height, shading indicates heating $\left(Q_{\mathrm{CAM}}\right.$ and $\left.Q_{\mathrm{SW}}\right)$, and vectors indicate the wind field. Contour interval is $0.6 \mathrm{~m}$ before transition and $3 \mathrm{~m}$ after, showing that the wave amplitude is increased by a factor of 5 .

departures from the zonal mean (e.g., $u^{\prime}=u-\bar{u}$ ). The agreement between CAM and theory appears to be quite good; the theory captures qualitative shifts in phase tilt and amplitude across the bifurcation.

The analytical solutions can be used to calculate a zonal-mean eddy momentum flux convergence on the equator $-\left.\partial_{y} \overline{\left(u^{\prime} v^{\prime}\right)}\right|_{\text {eqtr }}$ as a function of $U$. The resulting dependence is shown in Fig. 4a, along with a similar curve derived from a CAM simulation in which $Q_{0}$ was increased slowly and continuously $\left(0.03 \mathrm{~K} \mathrm{day}^{-1} \mathrm{yr}^{-1}\right)$, to allow the zonal wind and eddy fluxes to be in quasiequilibrium with the forcing. Both curves indicate a maximum eddy flux when the mean flow nears $16 \mathrm{~m} \mathrm{~s}^{-1}$, the phase speed of the $n=1$ Rossby wave for the equivalent depth of $250 \mathrm{~m}$ used here. Although there is a relative phase shift between $u$ and $v$ associated with changes in $U$, most of the change in momentum transport is due to the amplification of the wave fields.

To evaluate the accuracy with which this theory reproduces the behavior seen in CAM, we run a series of simulations in which we vary $k$ and $c_{f}$. If the resonance is indeed determined by the zonal wind speed relative to the forcing, then we expect the velocity at which momentum convergence is maximized to be $U_{e}=-c_{R}+c_{f}$. Figure $4 \mathrm{~b}$ summarizes the velocity of maximum eddy momentum convergence in this set of CAM simulations.
The simulated $U$ is plotted against the expected value of $U_{e}$, with the Rossby wave phase speed given by

$$
c_{R}=\frac{-\beta}{k^{2}+(2 n+1) \beta / \sqrt{g H}},
$$

where we use $n=1$ and $H=250 \mathrm{~m}$ for all cases. The agreement with theory is again quite good, leading us to conclude that the shallow water theory does indeed capture the essential mechanism of the feedback and bifurcation. Two points, from $k=4, c_{f}=0$ and $k=6$, $c_{f}=0$, lie more than two standard deviations from the theoretical curve. It is unclear why, since they are both near the center of the model parameter space sampled here, but we take a moment to speculate why the theoretical solution might break down. First, the jet produced in the GCM is not uniform, but roughly Gaussian in meridional and vertical extent. The resulting shear may distort the wave field, and also modify the background vorticity gradient on which the waves propagate. This, in turn, leads to a change in the free wave velocity and shifts the resonance location. Second, the Phlips and Gill solution assumes small $U$ and $k$, but the dropped terms are not strictly negligible in all cases shown here. Finally, the eddy velocities can exceed $5 \mathrm{~m} \mathrm{~s}^{-1}$, suggesting that nonlinearities may be significant. 

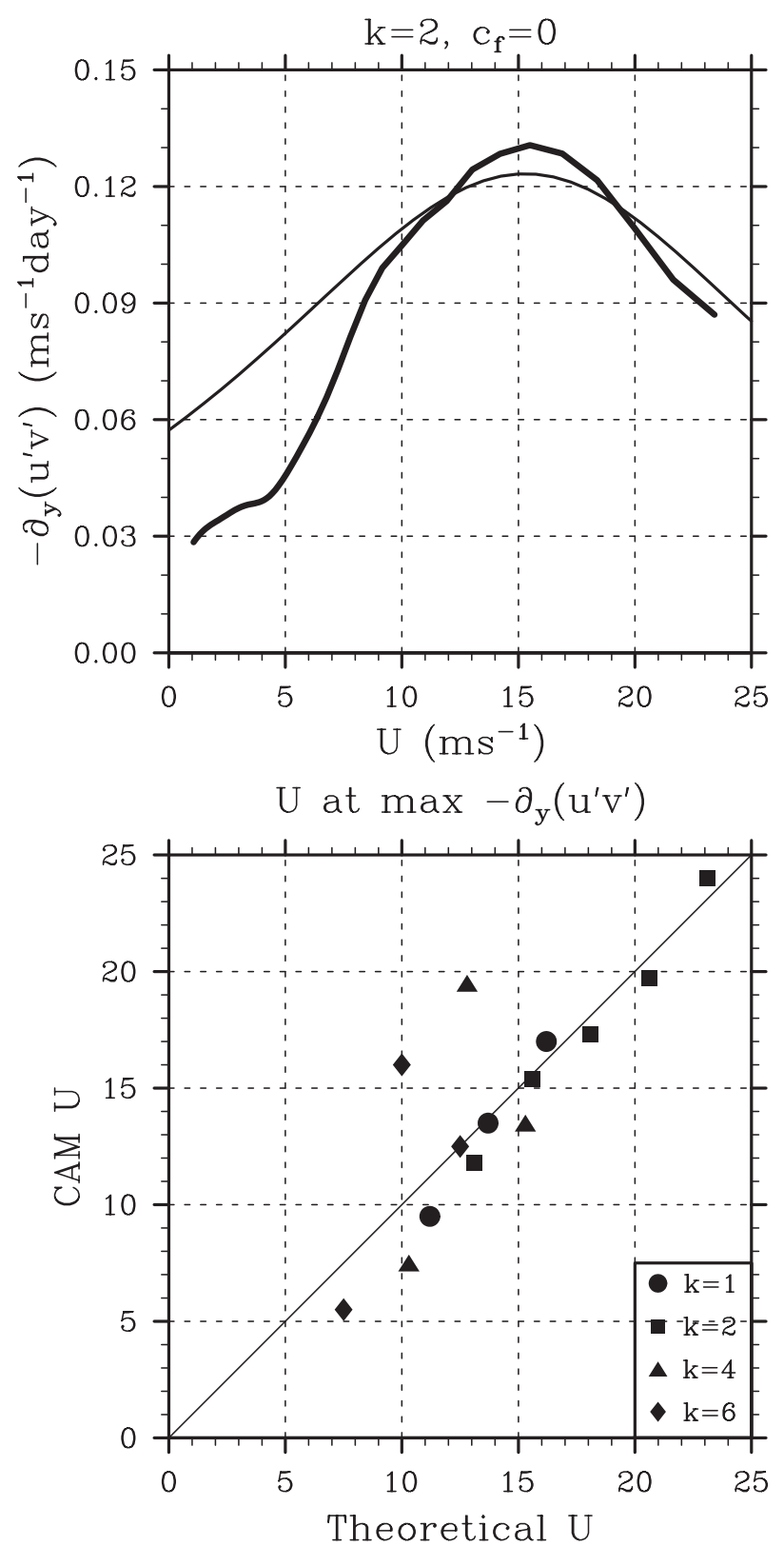

FIG. 4. (top) Equatorial momentum flux convergence as a function of jet velocity, in CAM with $\Delta T=0 \mathrm{~K}$ (thick line) and in shallow water theory (thin line). (bottom) Instantaneous jet velocity in CAM simulations at time of maximum eddy momentum convergence, plotted against the resonant velocity predicted by shallow water theory for the appropriate $k$ and $c_{f}$.

\section{The resonance in more realistic background states}

\section{a. Increasing $\Delta T$}

Having demonstrated that the resonance occurs in the idealized configuration with $\Delta T=0 \mathrm{~K}$, we now turn to more Earth-like simulations with the pole-to-equator temperature difference set to $\Delta T=40 \mathrm{~K}$. While this meridional temperature gradient is still less than on modern Earth, these simulations now include an equinoctial Hadley circulation and significant baroclinic eddy activity. Our goal is to demonstrate that the wave resonance and bifurcation are possible in a fully 3D atmosphere with a vigorous basic-state circulation. The zonal-mean zonal wind and meridional mass streamfunction from a run without asymmetric heating are shown in Fig. 5, along with climatological fields from the National Centers for Environmental Prediction (NCEP) reanalysis product (Kalnay et al. 1996). The strength of the midlatitude jets is comparable between our idealized CAM simulation and the reanalysis, although the jets in the idealized simulation extend to unrealistic altitudes. This is an artifact of the prescribed restoring temperature being uniform in the stratosphere. The Hadley cells are also significantly weaker in CAM because of the lack of offequatorial peak heating associated with a seasonal cycle, which is known to strengthen the winter hemisphere cell (e.g., Lindzen and Hou 1988). Despite these deficiencies, we believe this basic state is sufficiently realistic to make our point.

On top of this basic state we prescribe an equatorial heating crudely based on the MJO, with $k=1$ and $c_{f}=$ $5 \mathrm{~m} \mathrm{~s}^{-1}$. The results are qualitatively similar if we use the stationary heating with $k=2$ from section 3 , but a lower wavenumber proves to be advantageous, as we show in the next section.

We increase the heating rate in the stepwise manner described in section 3, generating the time series shown in Fig. 6. For $Q_{0}<0.5 \mathrm{~K} \mathrm{day}^{-1}$ there is little change in the upper tropospheric winds, which remain easterly, even at values of $Q_{0}$ that triggered strong superrotation when $\Delta T$ was set to $0 \mathrm{~K}$. The eddy momentum convergence rises according to the $Q_{0}^{2}$ scaling but is weaker than in the $\Delta T=0 \mathrm{~K}$ case, presumably because the equatorial eddy field is modified by baroclinic wave propagation in a way that reduces the velocity covariance.

At $Q_{0}=0.6 \mathrm{~K} \mathrm{day}^{-1}$ the mean wind become weakly westerly and the eddy momentum convergence begins to rise faster than the $Q_{0}^{2}$ scaling. This departure from linearity is expected as the mean wind approaches the resonance velocity. Increases in the eddy momentum convergence are still relatively small, however, and are quickly offset by a negative feedback involving momentum advection by the mean circulation $-\bar{\omega} \partial_{p} \bar{u}$, which prevents a runaway acceleration. This trend continues until $Q_{0}=0.9 \mathrm{~K}_{\text {day }}{ }^{-1}$ (compared with $Q_{0}=$ $0.4 \mathrm{~K}$ day $^{-1}$ for $\Delta T=0 \mathrm{~K}$ ), at which point the jet jumps from roughly 8 to $50 \mathrm{~m} \mathrm{~s}^{-1}$ and eventually settles to a statistical equilibrium of $45 \mathrm{~m} \mathrm{~s}^{-1}$ (compared with $24 \mathrm{~m} \mathrm{~s}^{-1}$ for $\left.\Delta T=0 \mathrm{~K}\right)$. 

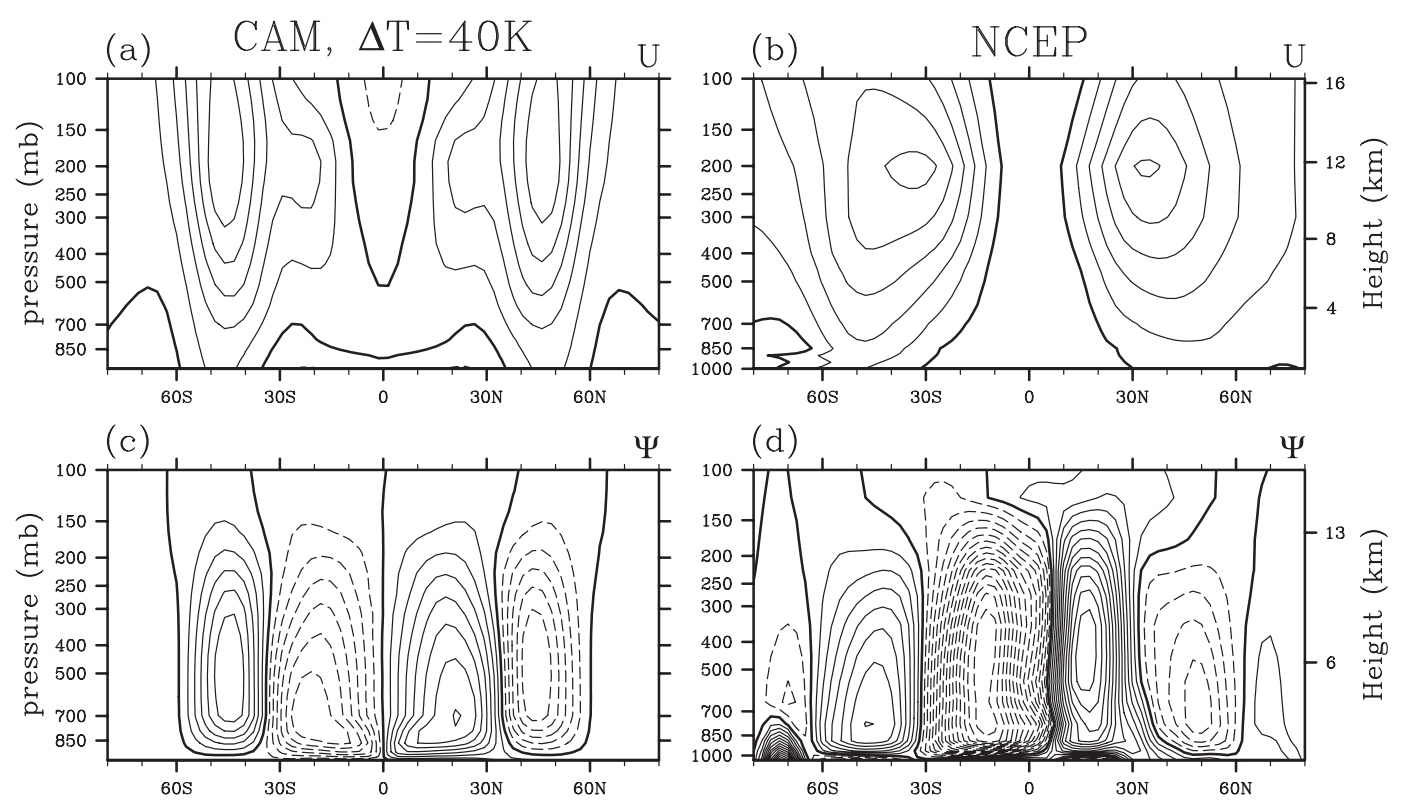

FIG. 5. Zonal- and time-mean fields from the (a),(c) CAM $\Delta T=40 \mathrm{~K}$ unforced simulation and (b),(d) NCEP reanalysis. Contour intervals are (a),(b) $5 \mathrm{~m} \mathrm{~s}^{-1}$ for zonal wind and (c),(d) $10 \times 10^{9} \mathrm{~kg} \mathrm{~s}^{-1}$ for the meridional overturning streamfunction.

This transition differs from the $\Delta T=0 \mathrm{~K}$ case in two respects. The equatorial jet is more than twice as strong here, arguably due to the larger heating rate at the point of bifurcation. That the transition occurs at a higher value of $Q_{0}(0.9 \mathrm{~K}$ instead of $0.4 \mathrm{~K})$ would suggest a factor of $5\left(0.9^{2} / 0.4^{2}\right)$ difference in momentum convergence. Of course, the resonant behavior renders this scaling inadequate across the bifurcation, and other terms in the momentum equation may compensate the equatorial eddy flux, but it is obvious that a larger $Q_{0}$ can account for much of the difference in postbifurcation jet velocity.

The transition here also differs qualitatively from the $\Delta T=0 \mathrm{~K}$ case. The postbifurcation circulation with $\Delta T=0 \mathrm{~K}$ looked like an approximately Gaussian jet superimposed on the basic state, but we find here that the background flow is significantly modified. The Hadley cell largely collapses after the transition (Fig. 7d), consistent with changes seen with $\Delta T=0 \mathrm{~K}$. More interesting is the equatorward shift of the midlatitude jets, such that they merge with the equatorial jet and produce an atmosphere with only westerly winds above $600 \mathrm{mb}$. This behavior was also noted by Held (1999) in a similar model with equatorial forcing.

\section{b. Comparing feedbacks}

Although the results presented above are reminiscent of the bifurcation in section 3 , it would be premature to conclude that the $\Delta T=40 \mathrm{~K}$ bifurcation is driven by the same wave resonance. The presence of a Hadley circulation and midlatitude jets brings into play the additional feedbacks identified in previous studies. In this section we hope to evaluate their relative contributions, if any, to the transition to superrotation.

We first consider the feedback involving midlatitude wave propagation identified by Saravanan (1993). Because the forced equatorial eddies have a wavenumber

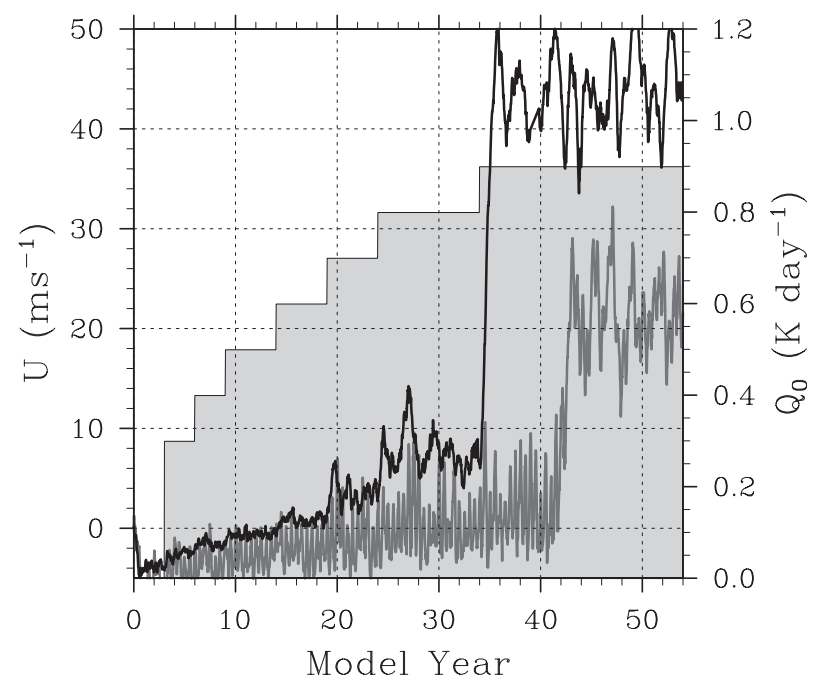

FIG. 6. Forcing amplitude $Q_{0}$ (piecewise-constant gray shading) and equatorial jet velocity (black line) for a CAM simulation with a more realistic pole-to-equator temperature difference of $T=$ $40 \mathrm{~K}$. Jet velocity for a simulation including a seasonal cycle (gray line) with the same forcing $Q_{0}$. 

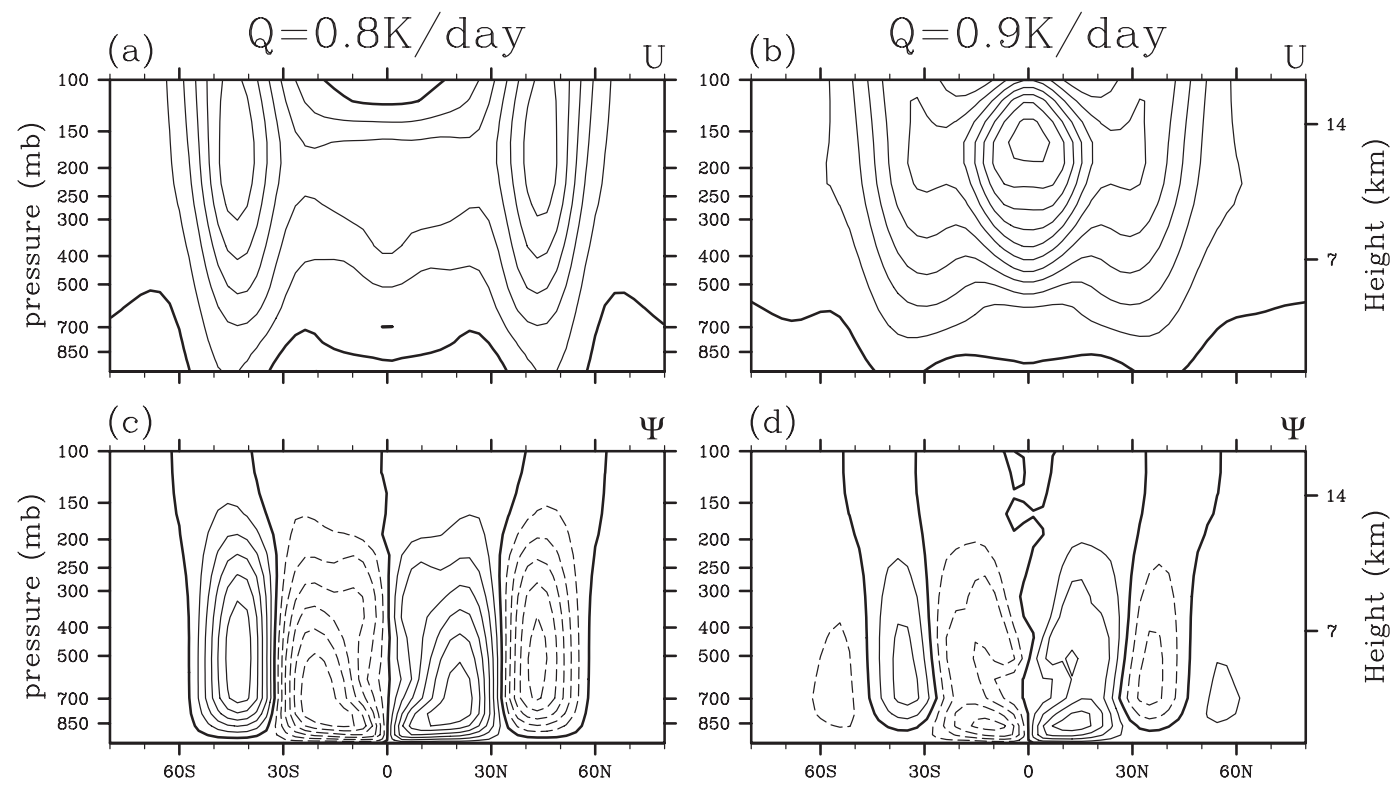

FIG. 7. Zonal- and time-mean fields for CAM simulations with equatorial heat forcing. Contours are as in Fig. 5.

$k=1$, while midlatitude baroclinic eddy energy peaks at $k=5$ and is largely negligible for $k<3$, we expect the momentum fluxes attributable to each source to be separable in wavenumber space. To this end, we calculate the momentum flux cospectrum

$$
\Phi=\frac{1}{N_{x}} \hat{u}^{\prime} * \hat{v}^{\prime}
$$

where $\hat{A}$ designates the Fourier transform of $A$ in longitude, $\hat{A}^{*}$ is the complex conjugate of $\hat{A}$, and $A^{\prime}$ is the instantaneous zonal anomaly $A^{\prime}=A-\bar{A}$. The $100-400-\mathrm{mb}$ zonal-mean cospectrum averaged over the final $2 \mathrm{yr}$ of the $Q_{0}=0.8 \mathrm{~K} \mathrm{day}^{-1}$ segment, just prior to the bifurcation, is shown in Fig. 8. The equatorial eddy $(k=1)$ and midlatitude eddy $(k>3)$ momentum fluxes are clearly separable and play very different roles in the momentum balance; the former is associated with strong momentum convergence on the equator, and the latter with a weak divergence, as expected.

Having established their separability, we now evaluate their relative fluxes during three segments of the simulation, with $Q_{0}=0,0.8$, and $0.9 \mathrm{~K} \mathrm{day}^{-1}$, corresponding to the unforced state and just before and just after the bifurcation, respectively. The zonal-mean eddy momentum flux associated with $k=1$ is shown in the left panels of Fig. 9, as a function of latitude and pressure. The flux is negligible without forcing, but with $Q_{0}=$ $0.8 \mathrm{~K} \mathrm{day}^{-1}$ a strong convergence is evident over the equator. In the right panels we show the total flux associated with higher wavenumbers (i.e., the summation of the cospectrum over $\left.k>1, \sum_{k=2}^{N_{k}} \Phi\right)$. The basic state shows a weak divergence over the tropics, and very little change when asymmetric heating is applied, indicating an absence of the wave feedbacks hypothesized by Saravanan (1993). Note from Fig. 7 that the upper

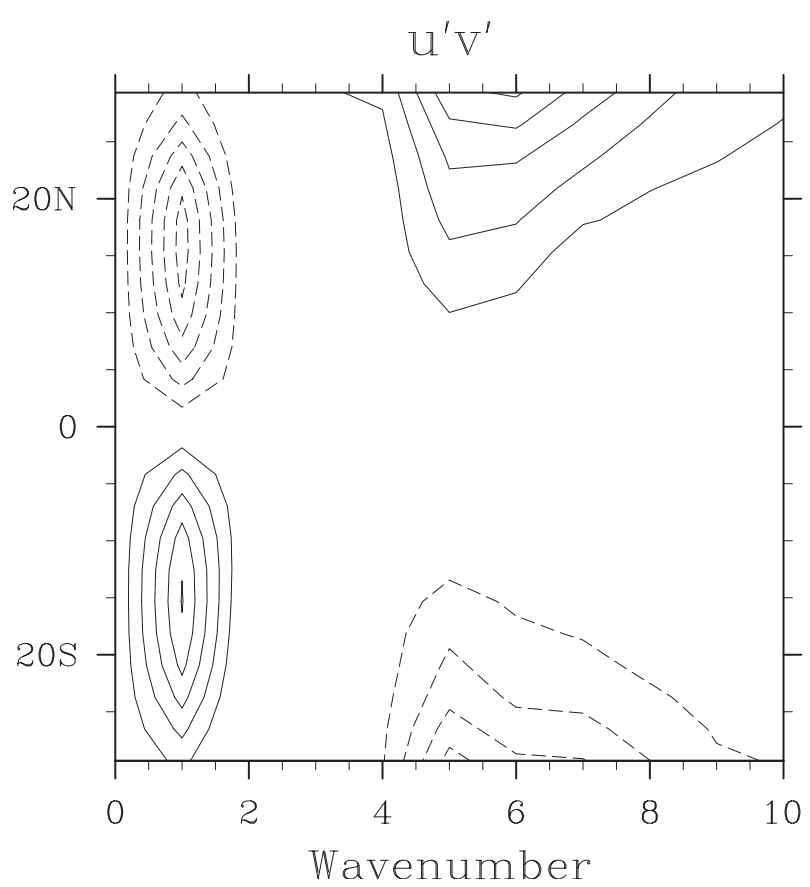

FIG. 8. Zonal- and time-mean eddy momentum flux cospectrum, integrated $100-400 \mathrm{mb}$. The separation of equatorial eddies $(k=1$, with flux toward equator) and midlatitude eddies $(k>3$, with flux away from equator) is clearly visible. 

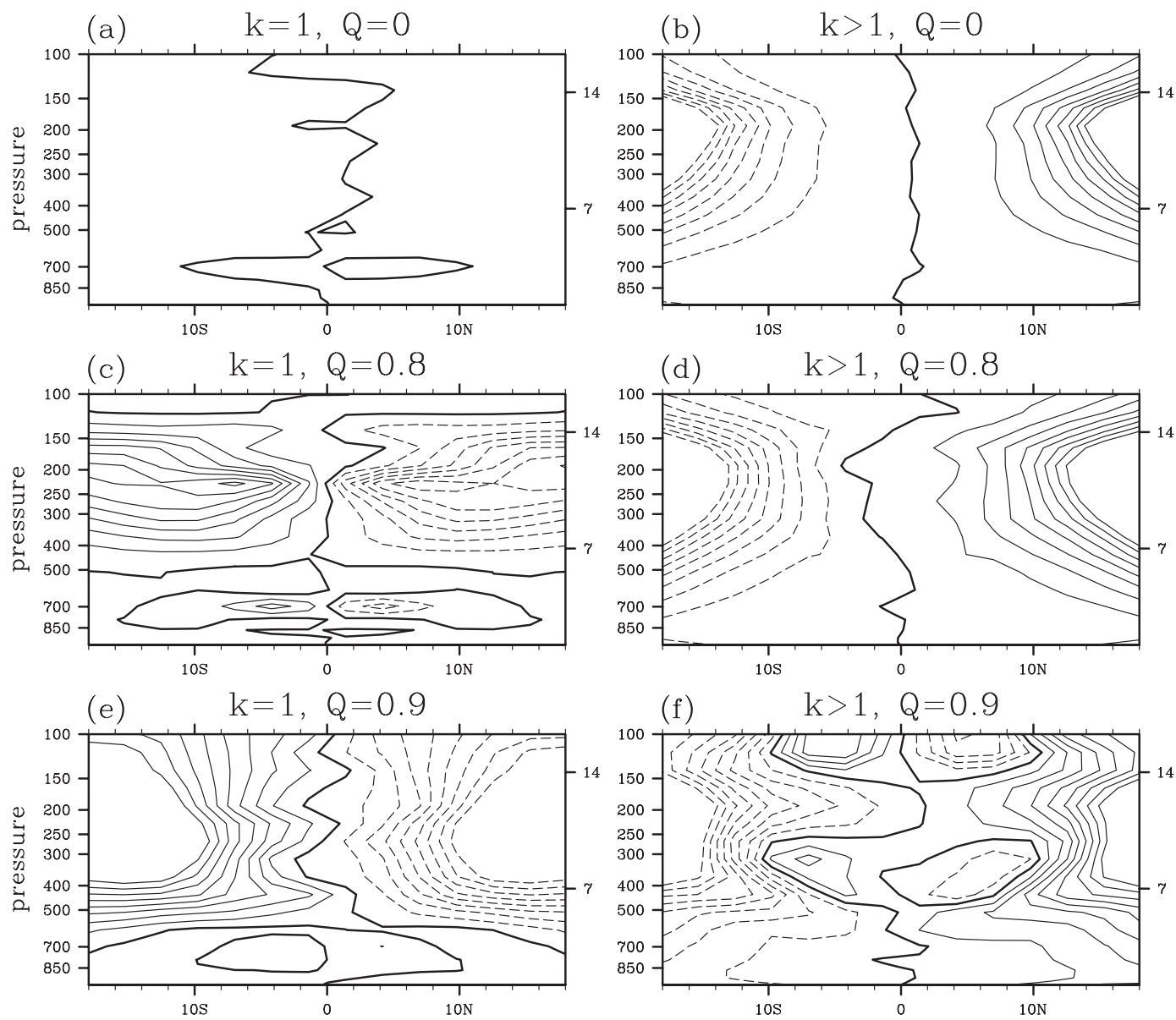

FIG. 9. Zonal-mean eddy momentum flux separated by zonal wavenumber, with (a),(c),(e) $k=1$ and (b),(d),(f) integrations over $k>1$. The heating rate $Q_{0}$ is indicated for each time period.

tropospheric equatorial zonal wind is already above $10 \mathrm{~m} \mathrm{~s}^{-1}$ when $Q_{0}=0.8 \mathrm{~K} \mathrm{day}^{-1}$, and the critical latitudes predicted by linear barotropic wave theory should have moved equatorward or disappeared for many wavenumbers. That the midlatitude momentum fluxes do not respond to changes in the background zonal wind suggests a failure of the linear theory.

After the bifurcation, there is an obvious amplification of the $k=1$ momentum flux convergence, while the $k>1$ fluxes become less spatially coherent (Fig. 9f), with equatorial convergence on some levels and divergence on others. We note that after the bifurcation, the Rayleigh criterion for barotropic instability (that the absolute vorticity $\beta-U_{y y}$ change sign in the domain) is satisfied on the poleward flanks of the equatorial jet, around $15^{\circ} \mathrm{N} / \mathrm{S}$ and between 200 and $400 \mathrm{mb}$. This suggests that the disorganized character of the $k>1$ momentum flux may be due to waves generated locally by barotropic instability interacting with the baroclinic waves propagating from midlatitudes.
We also consider the axisymmetric feedback proposed by Shell and Held (2004), which requires a reduction in vertical advection of quiescent surface air into the jet by the Hadley cell, as the equatorial winds accelerate. We use the vertical convergence of momentum $-\bar{\omega} \partial_{p} \bar{u}$ integrated over the jet, for comparison with their term $R$, which represents mass exchange between the upper and lower layers. The mean vertical convergence in this simulation is significantly smaller than the eddy flux convergences and almost certainly plays a minor role in the bifurcation.

\section{c. Including a seasonal cycle}

The largest source of easterly acceleration in the equatorial upper troposphere comes from the cross-equatorial flow associated with the solstitial Hadley circulation (Lee 1999), and we would be remiss not to address it here. To understand its effect on the bifurcation, we add a simple seasonal cycle to the thermodynamic tendency, of the form 


$$
\begin{aligned}
Q_{s}= & \left(1.0 \mathrm{~K} \mathrm{day}^{-1}\right) \exp \left[-\left(\frac{\phi-7.5^{\circ} \sin 2 \pi t / \tau}{7.5^{\circ}}\right)^{2}\right] \\
& -\left(0.5 \mathrm{~K} \mathrm{day}^{-1}\right) \exp \left[-\left(\frac{\phi-15^{\circ} \sin 2 \pi t / \tau}{15^{\circ}}\right)^{2}\right], \\
p_{t} & <p<p_{s}
\end{aligned}
$$

where $\phi$ is latitude, $t$ is time, and $\tau$ is $1 \mathrm{yr}$. This is a timedependent version of the stationary off-equatorial heating prescribed by Kraucunas and Hartmann (2005). In addition, we multiply $Q_{s}$ by the factor $\Delta T / 60 \mathrm{~K}$, such that the seasonal cycle scales with the meridional temperature gradient. The $T_{s}$ term leads to a vigorous winter hemisphere Hadley cell and more realistic equatorial momentum balance (not shown).

We again apply the $k=1$ equatorial heating from the last section, generating the time series in Fig. 6. A bifurcation is clearly visible at year 42 , as the mean equatorial winds between 100 and $400 \mathrm{mb}$ abruptly increase by $20 \mathrm{~m} \mathrm{~s}^{-1}$ and become strongly superrotating. This occurs at the same heating rate required for the equinoctial case $\left(Q_{0}=0.9 \mathrm{~K} \mathrm{day}^{-1}\right)$, but the jump in velocity is about $10 \mathrm{~m} \mathrm{~s}^{-1}$ smaller. Interestingly, the model does not immediately transition to strong superrotation when $Q_{0}$ is set to $0.9 \mathrm{~K} \mathrm{day}^{-1}$ but rather maintains weak westerlies for $8 \mathrm{yr}$ before making a seemingly spontaneous jump. A 20-yr extension of the simulation (not shown) in which the jet remains near $20 \mathrm{~m} \mathrm{~s}^{-1}$ suggests that the upper equilibrium is fully stable. Similar extensions in other simulations with various $\Delta T$ and $Q_{0}$ suggest that most values of $Q_{0}$ have a single stable fixed point, either strongly superrotating or not. The only exceptions occur at the boundary of the two regimes, where seemingly spontaneous jumps are possible, although they only go one way (the strongly superrotating equilibrium is always stable). We attribute this apparent spontaneity to the stochastic influence of midlatitude eddies, which leads to variation in both the eddy momentum flux out of the tropics, and the coherence of the forced equatorial wave. This provides temporary windows of opportunity for positive feedbacks to take hold and produce strong superrotation. The seasonal cycle would add a periodic component to this variability but could not itself account for a multiyear delay.

\section{Summary and conclusions}

Superrotation in several atmospheric GCMs has coincided with significant increases in organized tropical convection with gross characteristics resembling the Madden-Julian oscillation (Lee 1999; Caballero and Huber 2010). Organized convection is believed to excite atmospheric Rossby waves that lead to equatorward momentum fluxes and drive the atmosphere toward a superrotating state. These increases in convective variability often occur in simulations of warmer climates, which suggests the possibility of superrotation during past warm intervals (Tziperman and Farrell 2009) or a future dominated by anthropogenic warming (Held 1999; Pierrehumbert 2000).

In this study, we mimic the dynamical effects of organized tropical convection by applying a zonally varying equatorial heating in a GCM with simplified physics, resulting in eddy momentum fluxes from the subtropics to the equator. A comparison with analytic shallow water theory suggests that the equatorward momentum flux is produced by the tilted planetary-scale Rossby gyres of the classical Matsuno-Gill response to imposed heating. This is similar to the numerical shallow water results of Showman and Polvani (2010), as well as studies of transient superrotation associated with the MJO (e.g., Biello et al. 2007; Moncrieff 2004). We find that with sufficient equatorial forcing, these fluxes produce a strong westerly flow between 200 and $400 \mathrm{mb}$-in essence, a "superrotating jet."

The strength of the superrotating jet was shown to depend nonlinearly on the equatorial heating rate $Q_{0}$ and the pole-to-equator temperature difference $\Delta T$. When $\Delta T=0 \mathrm{~K}$, both the Hadley circulation and baroclinic wave activity are eliminated. In this simplified regime, the superrotating jet undergoes a bifurcation as $Q_{0}$ passes from 0.3 to $0.4 \mathrm{~K} \mathrm{day}^{-1}$, abruptly transitioning from a relatively weak $\left(<5 \mathrm{~m} \mathrm{~s}^{-1}\right)$ to a strong $\left(24 \mathrm{~m} \mathrm{~s}^{-1}\right)$ state. If the heating rate is reduced after the bifurcation occurs, the jet remains in the strong state. This hysteresis is moderate, however; multiple equilibria are found only for $0.25<Q_{0}<0.35 \mathrm{~K} \mathrm{day}^{-1}$.

Distinct weak and strong states are still observed with a nonzero meridional temperature gradient, although the presence of a vigorous meridional circulation and baroclinic wave activity increases the value of $Q_{0}$ required to force a transition. For example, with $\Delta T=$ $40 \mathrm{~K}$, the jet will jump from 10 to $45 \mathrm{~m} \mathrm{~s}^{-1}$ as $Q_{0}$ passes from 0.8 to $0.9 \mathrm{~K} \mathrm{day}^{-1}$. Hysteresis is no longer seen, likely due to the increased variability in the simulated atmosphere. The transition to superrotation in this case includes a nearly $50 \%$ reduction in the Hadley circulation strength and an equatorward shift of the midlatitude jets, which effectively merge with the equatorial jet. Shifts in surface winds associated with the midlatitude jets may help explain sea surface temperature anomalies at midlatitude upwelling sites in the early Pliocene (Tziperman and Farrell 2009).

Kraucunas and Hartmann (2005) suggested that the sensitivity to equatorial eddy heating in idealized models is an artifact of their lack of a seasonal cycle, especially 
the solstitial seasons of the Hadley circulation, which induce a strong easterly acceleration in the equatorial upper troposphere. We address this deficiency by adding an idealized seasonal forcing in the form of a zonally uniform heating tendency that migrates between hemispheres with an annual period. This produces a Hadley circulation dominated by the "winter" hemisphere and results in much stronger equatorial easterlies, consistent with observations (Lee 1999). Despite this change, a bifurcation and transition to a strongly superrotating jet are still seen for $\Delta T=40 \mathrm{~K}$, although the "strong" state westerlies are weaker than in the equinoctial simulation.

The nonlinear response to heating reported here is reminiscent of behavior seen in two-layer primitive equation models (Suarez and Duffy 1992; Saravanan 1993), and the axisymmetric model of Shell and Held (2004), but the mechanism responsible for our bifurcation is a novel one and has not previously appeared in the superrotation literature. Instead of feedbacks involving midlatitude waves or the Hadley circulation, our bifurcation is driven by a resonance phenomenon. As the zonal wind relative to the imposed heating nears the phase speed of the $n=1$ free equatorial Rossby wave, the heating projects onto the free wave mode and the overall Rossby wave response amplifies. The amplified wave produces additional momentum flux from the subtropics to the equator, which ultimately allows a higher equilibrium jet velocity. This resonance is directly analogous to the theoretical singularity involving free topographic Rossby waves in the presence of a westerly mean wind (cf. Holton 2004).

The difference in mechanisms helps explain why the hysteresis in our model is so much weaker than that reported by Saravanan (1993). The superrotating state in that model was supported by transient eddies-rather than the forced stationary eddies in our simulationswhich allowed the superrotation to persist for some time after heating was removed. We also find little evidence for the midlatitude wave feedbacks found in Saravanan's simulations. This is consistent with previous studies using multilevel GCMs (e.g., Hoskins et al. 1999) and analytical work that indicates that Rossby wave breaking and its associated momentum convergence may depend on the vertical shear. Panetta et al. (1987) found that in the twolayer quasigeostrophic framework, Rossby waves propagating into a region where $U-c$ is positive in the upper layer and negative in the lower layer will not exhibit critical layer absorption when the vertical shear exceeds a critical value. This perfect transparency does not seem to occur in the continuous case (Held 1999), which suggests that the dramatic positive feedback found in two-layer models may be an artifact of their vertical resolution. This feedback was invoked by Mitchell and Vallis (2010) to explain transitions to superrotation in multilevel simulations of generic terrestrial planets, but it is unclear why the wave feedback would occur in that context but not here.

The resonance presented here joins other feedbacks (Williams 2003; Shell and Held 2004) that provide plausible mechanisms for abrupt, qualitative changes in the large-scale flow of three-dimensional atmospheres. Simulations most similar to modern Earth, with $\Delta T=$ $60 \mathrm{~K}$ (not shown), indicate a much more linear response to changes in $Q_{0}$ and little evidence of abrupt transition, suggesting that a resonance-driven bifurcation may be limited to past or potential future climates in which $\Delta T$ or the seasonal cycle are reduced. The possibility of superrotation in the larger context of Earth history is only beginning to be understood.

Acknowledgments. The authors thank Zhiming Kuang for many helpful discussions, Chris Walker for invaluable assistance with modifications to CAM, and Isaac Held and one anonymous reviewer for their comments. This work was partially supported by a graduate student fellowship from the National Science Foundation (NA), by NSF Climate Dynamics Grants ATM-0902844 and ATM-0754332 (ET), and by NSF ATM-0736022 (BF). ET thanks the Weizmann Institute for its hospitality during parts of this work.

\section{APPENDIX}

\section{Derivation of the Shallow Water Solutions}

Phlips and Gill (1987) found analytic solutions for the equatorial response to a heating of limited spatial extent. We consider the simplified problem of a global-scale sinusoidal heating of the form $Q=Q_{0} \cos (k x) \exp \left(-y^{2} / 4\right)$. It is assumed that the solutions are separable in $x, y$, and $z$, and that the heating projects only onto the first baroclinic mode. This allows variables to be expressed as, for example, $Q=F(x) \Sigma Q_{n} D_{n}(y)$, where $D_{n}(y)$ is the $n$th parabolic cylinder function. We refer the reader to the original paper for additional details.

We nondimensionalize the shallow water equations, using horizontal scale $\sqrt{c / 2 \beta}$ and temporal scale $(2 \beta c)^{1 / 2}$ :

$$
\begin{aligned}
\partial_{t} u+U \partial_{x} u+\epsilon u-\beta y v & =-\partial_{x} \phi, \\
\partial_{t} v+U \partial_{x} v+\epsilon v-\beta y u & =-\partial_{y} \phi, \\
\partial_{t} \theta+U \partial_{x} \theta+\epsilon \theta+w & =Q, \\
\theta & =\partial_{z} \phi, \\
\partial_{x} u+\partial_{y} v+\partial_{z} w & =0 .
\end{aligned}
$$


Defining $q=\phi+u$ and $r=\phi-u$, it can be shown that the steady-state Kelvin mode response is governed by the single equation

$$
\left(U \partial_{x}+\epsilon\right) q_{0}+\partial_{x} q_{0}=-Q_{0} \cos (k x) .
$$

Substituting an assumed solution of the form $q_{0}=$ $A \cos (k x)+B \sin (k x)$ leads to $A=-Q_{0} \gamma^{2} / \epsilon\left(1+\gamma^{2}\right)$ and $B=-Q_{0} \gamma / \epsilon\left(1+\gamma^{2}\right)$, where $\gamma=\epsilon / k\left(U+c_{n}\right)$ and $c_{n}$ is the wave speed for mode $n$.

Relating $q$ to $u$ and $\phi$, we find

$u_{k}=\phi_{k}=\frac{-Q_{0} \gamma}{2 \epsilon\left(1+\gamma^{2}\right)}[\gamma \cos (k x)+\sin (k x)] \exp \left(-y^{2} / 4\right)$,

where the subscript $k$ designates the Kelvin mode response.

The Rossby modes $n>0$ are governed by three equations, after dropping terms that are second order in $\epsilon$ and $U$ :

$$
\begin{aligned}
(U k+\epsilon) q_{n+1}+k q_{n+1}-v_{n} & =-Q_{n+1} F(x), \\
(U k+\epsilon) r_{n-1}-k r_{n-1}+n v_{n} & =Q_{n-1} F(x), \\
2(U k+\epsilon) v_{n}+(n+1) q_{n+1}-r_{n-1} & =0 .
\end{aligned}
$$

These can be manipulated to find a single equation in $q$. Since our forcing $Q$ projects only onto the $n=1$ mode, this equation is given by

$$
(1-3 U) k q_{2}-3 \epsilon q_{2}=Q_{0} \cos (k x)
$$

Substituting a solution of the form $q_{2}=A \cos (k x)+$ $B$ in $(k x)$ leads to $A=-(1 / 3) Q_{0} \gamma^{2} / \epsilon\left(1+\gamma^{2}\right)$ and $B=$ $-(1 / 3) Q_{0} \gamma / \epsilon\left(1+\gamma^{2}\right)$.

Finally, $q_{2}$ may be translated into $u$ and $v$ :

$$
\begin{aligned}
u= & \frac{Q_{0} \gamma}{6 \epsilon\left(1+\gamma^{2}\right)}[\gamma \cos (k x)+\sin (k x)]\left(3-y^{2}\right) \exp \left(-y^{2} / 4\right) \\
v= & \left\{\frac{-(4 / 3) Q_{0} \gamma}{\epsilon\left(1+\gamma^{2}\right)}[U k+\epsilon \gamma \cos (k x)+(\epsilon-U k \gamma) \sin (k x)]\right. \\
& \left.+Q_{0} \cos (k x)\right\} y \exp \left(-y^{2} / 4\right)
\end{aligned}
$$

\section{REFERENCES}

Biello, J. A., A. J. Majda, and M. W. Moncrieff, 2007: Meridional momentum flux and superrotation in the multiscale IPESD MJO model. J. Atmos. Sci., 64, 1636-1651.

Caballero, R., and M. Huber, 2010: Spontaneous transition to superrotation in warm climates simulated by CAM3. Geophys. Res. Lett., 37, L11701, doi:10.1029/2010GL043468.

Dima, I. M., J. M. Wallace, and I. Kraucunas, 2005: Tropical zonal momentum balance in the NCEP reanalyses. J. Atmos. Sci., 62, 2499-2513.
Gill, A. E., 1980: Some simple solutions for heat-induced tropical circulation. Quart. J. Roy. Meteor. Soc., 106, 447-462.

Grabowski, W. W., 2004: An improved framework for superparameterization. J. Atmos. Sci., 61, 1940-1952.

Held, I. M., 1999: Equatorial superrotation in Earth-like atmospheric models. Berhard Haurwitz Memorial Lecture, presented at the Annual Meeting of the American Meteorological Society, Dallas, TX. [Available online at http://www.gfdl.noaa.gov/ cms-filesystem-action/user_files/ih/lectures/super.pdf.]

_, and M. J. Suarez, 1978: A two-level primitive equation atmospheric model designed for climatic sensitivity experiments. J. Atmos. Sci., 35, 206-229.

, and - 1994: A proposal for the intercomparison of the dynamical cores of atmospheric general circulation models. Bull. Amer. Meteor. Soc., 75, 1825-1830.

Hide, R., 1969: Dynamics of the atmospheres of the major planets with an appendix on the viscous boundary layer at the rigid bounding surface of an electrically conducting rotating fluid in the presence of a magnetic field. J. Atmos. Sci., 26, $841-852$.

Holton, J. R., 2004: An Introduction to Dynamic Meteorology. 4th ed. Elsevier, 511 pp.

Hoskins, B. J., R. B. Neale, M. Rodwell, and Y. Gui-Ying, 1999: Aspects of the large-scale tropical atmospheric circulation. Tellus, 51, 33-44.

Huang, H.-P., P. Sardeshmukh, and K. Weickmann, 1999: The balance of global angular momentum in a long-term atmospheric data set. J. Geophys. Res., 104, 2031-2040.

—, K. M. Weickmann, and C. J. Hsu, 2001: Trend in atmospheric angular momentum in a transient climate change simulation with greenhouse gas and aerosol forcing. J. Climate, 14, 1525-1534.

Kalnay, E., and Coauthors, 1996: The NCEP/NCAR 40-Year Reanalysis Project. Bull. Amer. Meteor. Soc., 77, 437-471.

Kiladis, G. N., K. H. Straub, and P. T. Haertel, 2005: Zonal and vertical structure of the Madden-Julian oscillation. J. Atmos. Sci., 62, 2790-2809.

Kraucunas, I., and D. L. Hartmann, 2005: Equatorial superrotation and the factors controlling the zonal-mean zonal winds in the tropical upper troposphere. J. Atmos. Sci., 62, 371-389.

Lee, S., 1999: Why are the climatological zonal winds easterly in the equatorial upper troposphere? J. Atmos. Sci., 56, 13531363.

Lindzen, R. S., and A. Y. Hou, 1988: Hadley circulations for zonally averaged heating centered off the equator. J. Atmos. Sci., 45, 2416-2427.

Mitchell, J. L., and G. K. Vallis, 2010: The transition to superrotation in terrestrial atmospheres. J. Geophys. Res., 115, E12008, doi:10.1029/2010JE003587.

Moncrieff, M. W., 2004: Analytic representation of the large-scale organization of tropical convection. J. Atmos. Sci., 61, 15211538.

Panetta, R. L., I. Held, and R. Pierrehumbert, 1987: External Rossby waves in the two-layer model. J. Atmos. Sci., 44, 2924 2933.

Phlips, P., and A. Gill, 1987: An analytic model of the heat-induced tropical circulation in the presence of a mean wind. Quart. J. Roy. Meteor. Soc., 113, 213-236.

Pierrehumbert, R. T., 2000: Climate change and the tropical Pacific: The sleeping dragon wakes. Proc. Natl. Acad. Sci. USA, 97, 1355-1358.

Randel, W. J., and I. M. Held, 1991: Phase speed spectra of transient eddy fluxes and critical layer absorption. J. Atmos. Sci., 48, 688-698. 
Salby, M. L., and R. R. Garcia, 1987: Transient response to localized episodic heating in the tropics. Part I: Excitation and short-time near-field behavior. J. Atmos. Sci., 44, 458-498.

Saravanan, R., 1993: Equatorial superrotation and maintenance of the general circulation in two-level models. J. Atmos. Sci., 50, 1211-1227.

Schumacher, C., R. Houze Jr., and I. Kraucunas, 2004: The tropical dynamical response to latent heating estimates derived from the TRMM precipitation radar. J. Atmos. Sci., 61, 1341-1358.

Shell, K. M., and I. M. Held, 2004: Abrupt transition to strong superrotation in an axisymmetric model of the upper troposphere. J. Atmos. Sci., 61, 2928-2936.
Showman, A. P., and L. M. Polvani, 2010: The Matsuno-Gill model and equatorial superrotation. Geophys. Res. Lett., 37, L18811, doi:10.1029/2010GL044343.

Suarez, M. J., and D. G. Duffy, 1992: Terrestrial superrotation: A bifurcation of the general circulation. J. Atmos. Sci., 49, 15411554.

Tziperman, E., and B. Farrell, 2009: Pliocene equatorial temperature: Lessons from atmospheric superrotation. Paleoceanography, 24, PA1101, doi:10.1029/2008PA001652.

Williams, G. P., 2003: Barotropic instability and equatorial superrotation. J. Atmos. Sci., 60, 2136-2152.

2006: Equatorial superrotation and barotropic instability: Static stability variants. J. Atmos. Sci., 63, 1548-1557. 